\title{
Problemas y contradicciones de las políticas de conciliación trabajo-familia: análisis y propuestas desde la sociología relacional
}

\author{
Ángela Corró Tormo \\ Universitat Jaume I \\ angela.corro@uji.es \\ Pablo García Ruiz \\ Universidad de Zaragoza \\ pgruiz@unizar.es
}

Resumen: En este artículo se analiza el problema de la conciliación de la vida familiar y la laboral, uno de los aspectos esenciales y determinantes del bienestar personal y familiar. El análisis tiene en cuenta la perspectiva de las familias, de las empresas, del sistema politico y de la cultura de la sociedad. En el trabajo se pone de manifiesto la necesidad de un enfoque relacional ( $y$ no meramente funcional) en las politicas actuales que abordan el tema de la conciliación. Este enfoque relacional, explícito en la obra del sociólogo italiano Pierpaolo Donati, resulta a nuestro juicio más adecuado a las necesidades reales de la conciliación.

Palabras clave: conciliación familia-trabajo, politicas sociolaborales.

Abstract: This article analyzes the problem of reconciling work and family life, one of the key issues of personal and family welfare and wellbeing. The analysis is approached from the perspective of families, businesses, the political system and culture. The paper highlights the lack of a relational approach (not merely functional) in the current policies that address the issue of reconciliation. Within this specific sociological approach, the proposal of the Italian sociologist Pierpaolo Donati addresses the real needs of reconciliation.

Keywords: reconciliation work and family, social policies. 


\section{La conciliación como problema}

En los últimos veinte años se han sucedido las iniciativas políticas, empresariales y sociales para «solucionar» el problema de la conciliación entre la vida laboral y la familiar. Sin embargo, lejos de alcanzar una situación satisfactoria, las quejas y preocupaciones se mantienen o aumentan; de hecho, las dimensiones del problema parecen multiplicarse, pues lo que comenzó como un problema específico del ámbito laboral incluye hoy aspectos culturales, educativos, económicos, etc. Lejos de encontrar soluciones apropiadas, parece que nos encontramos con nuevos y más complejos problemas, e incluso con contradicciones y paradojas, en el desarrollo y la implantación de las políticas que pretenden ayudar a las personas a compatibilizar las exigentes condiciones del mercado laboral con las circunstancias cambiantes, y con frecuencia más difíciles, del ámbito familiar. En realidad, el problema de la conciliación depende del punto de vista desde el que se observe, de las estrategias que se pongan en marcha y de los objetivos primordiales que tales estrategias persigan.

\subsection{La conciliación como problema para las familias}

Desde el punto de vista de las familias, el problema de la conciliación aparece principalmente como un problema de tiempo, de estrés y de percibida o real incompatibilidad entre las exigencias del trabajo profesional y las necesidades de la realidad familiar (OECD, 2007; López y Acereda, 2007; Tobío, 2005). En efecto, las dificultades y aspectos problemáticos más frecuentemente mencionados son los siguientes: la escasez de tiempo para cuidar y educar a los hijos, la escasez de tiempo para atender a las personas mayores y dependientes a cargo, la escasez de tiempo para atender las tareas del hogar, el aumento del estrés como consecuencia de la dificultad para atender a estas expectativas, el incremento de la tensión y los conflictos familiares como consecuencia del estrés acumulado y, también, el aumento de enfermedades físicas y psíquicas derivadas de estas situaciones de dificultad y estrés.

En la competitiva sociedad actual, las familias experimentan dificultades para hacer compatibles los requerimientos del ámbito laboral con las aspiraciones del propio proyecto de vida (INE, 2011; Consejería de Empleo de Madrid, 2005). Esta situación se hace más aguda en determinadas circunstancias. Según el INE, en España, en 2010, diez millones de personas tenían al menos un hijo menor de 14 años; de ellas, un millón y medio utilizó servicios de cuidado para atenderles. En el mismo año, casi 850.000 personas no trabajaron, y no buscaron empleo - o trabajaron a tiempo parcial — para hacerse cargo regularmente de la 
atención a sus hijos. El 60\% de estas personas tomaron esta decisión porque los servicios de cuidado (guarderías, actividades extraescolares, etc.) eran «muy caros». Además, otras 290.000 personas no trabajaron ni buscaron empleo o lo hicieron a tiempo parcial para hacerse cargo regularmente de la atención a personas dependientes. El 95\% de estas personas que redujeron su dedicación al empleo para atender necesidades familiares fueron mujeres. En España, también según el INE, en 2010, 2.670.000 personas de entre 16 y 64 años se hacían cargo regularmente de la atención a personas mayores dependientes. De estas, 1.440 .000 estaban ocupadas en el mercado de trabajo. Las necesidades de conciliación son evidentes pero las dificultades también: el 27,5\% de los asalariados declaran no haber podido modificar su horario laboral por motivos familiares y el 31,5\% dice que no consiguió organizar su jornada para tomarse días libres por motivos familiares (INE, 2010).

\subsection{La conciliación como problema empresarial}

Desde el punto de vista de las empresas, la conciliación entre la vida laboral y familiar es también un problema importante (Chinchilla, 2007; Meil, García, Luque y Ayuso, 2010). Para las empresas, las exigencias de la conciliación se manifiestan de diversas formas. Entre ellas se cuenta, en primer lugar, el elevado absentismo por asuntos familiares. También se menciona con frecuencia el menor rendimiento o productividad por preocupaciones relacionadas con la propia familia (que incluyen la atención a menores y dependientes pero también la necesidad de gestionar los imprevistos, los problemas de educación o de salud, la tensión y el estrés, o incluso la separación o el divorcio). Desde un punto de vista organizativo, surge la necesidad de sustituir a las personas que tienen permisos maternales o paternales, o que están de baja puntual o relativamente prolongada por otros asuntos familiares, como enfermedades inesperadas de un hijo pequeño, u otros imprevistos en la atención de las obligaciones del hogar.

Para las empresas, la conciliación aparece primeramente como un problema de rendimiento y organización de las personas que deben atender a asuntos distintos del empleo, pero pronto surgen también otras dimensiones (EBTP, 2009). Si la empresa desea atraer o retener a personas particularmente cualificadas o valiosas deberá atender a sus demandas de conciliación (Albert, Escot, Fernández y Palomo, 2010). Con ello puede contentar a aquellos pero entonces deberá gestionar el probable descontento de quienes no se benefician de tales medidas e incluso terminan por cubrir o compensar de alguna forma las ausencias de quienes se acogen a las posibles medidas de conciliación. Un reto añadido es el de gestionar las posibles medidas de flexibilidad en el contexto de la negociación co- 
lectiva de las condiciones laborales en términos de igualdad de trato y de equidad para todos los trabajadores (Montoya i Barberá, 2007).

\subsection{La conciliación como problema político}

Desde los poderes públicos el reto de la conciliación se percibe también como un problema, pero con dimensiones y prioridades distintas. Los gobiernos son cada vez más conscientes de que deben atender cuestiones relacionadas con la conciliación trabajo-familia que exceden la capacidad (o el interés) de las empresas o las propias familias (Cardona, 2009; Hein, 2006) y que entran a formar parte de los sistemas de bienestar social (Campillo, 2010; Donati, 2007).

Por una parte, es motivo de preocupación la escasa tasa de natalidad, situada desde hace tiempo por debajo del límite de reemplazo generacional (Eurofound, 2010). Como es sabido, en las encuestas que se ocupan de este tema aparece repetidamente el hecho de que las familias desearían tener un número de hijos mayor que el que de hecho tienen, pero - entre otras razones - las condiciones del mercado laboral y la imposibilidad de conciliarlas con sus aspiraciones familiares se lo impiden. Desde el punto de vista del gobierno, la escasa natalidad se valora, sobre todo, por las consecuencias que la siguen en términos de envejecimiento de la población, y por tanto, en la presión sobre la sostenibilidad del sistema de pensiones, el gasto sanitario y la seguridad social que demanda un sociedad envejecida.

Por otra parte, los poderes públicos se plantean la necesidad de garantizar la atención de los dependientes (niños, mayores y personas con discapacidad) como un problema que excede las actuales capacidades de las familias, en particular las de «dos sustentadores», implicadas en mucho mayor medida en el mercado de trabajo. Además, también generan inquietud entre los responsables políticos otros problemas, como el incremento del fracaso escolar, que se derivan de una menor atención familiar a las necesidades educativas de los niños y adolescentes. En la medida en que la administración pública se ha ido ocupando progresivamente de estas inquietudes y demandas sociales, el problema de la conciliación se ha convertido también en un problema político.

\subsection{La conciliación como problema cultural}

La cuestión de la conciliación trabajo-familia contiene y refleja también un problema cultural (Torns, 2011). El modo en que se entienden y se atienden las necesidades de conciliación tiene una estrecha relación con el modo en que se entienden la igualdad y la corresponsabilidad en las relaciones familiares, muy en 
particular, en la distribución de tareas en el hogar y en la atención de las obligaciones familiares.

Se ha puesto de relieve con frecuencia que estamos en un momento de transición entre el modelo del varón bread-winner y el modelo de familia de dos sustentadores. Idealmente este segundo modelo ha sustituido al anterior, pero en la práctica los datos indican lo contrario. En concreto, los datos sobre el uso del tiempo y la dedicación a las tareas de hogar indican una gran diferencia entre varones y mujeres. (INE, 2011; Eurostat, 2005). Como hemos mencionado arriba, son también las mujeres las que mayoritariamente cuidan de los menores y las personas dependientes. Por estas razones, son también las mujeres las que tienen en mayor medida ocupaciones a tiempo parcial y las que soportan habitualmente la carga de la conciliación, hasta el punto de que se ha podido calificar la conciliación como un "problema femenino». Esta es la situación que se denuncia hoy en términos culturales como un anacronismo que se debe superar (Tobío, 2005). Se abre camino el término «corresponsabilidad» como denominación de un modelo de distribución del tiempo y de la dedicación al trabajo y a la familia más igualitario entre varones y mujeres. Sin embargo, este modelo no deja de ser contestado desde posiciones feministas que prefieren enfatizar la autonomía de la mujer más que su interdependencia, aunque sea en términos de igualdad.

Desde este punto de vista resulta patente que el problema de la conciliación consiste en que en las últimas décadas las mujeres se han incorporado al mercado de trabajo pero han continuado ocupándose también de las obligaciones de la vida familiar $y$, como consecuencia, se ven en la tesitura de aceptar empleos de inferior categoría, remuneración o posibilidades de carrera profesional, con lo que se perpetúan las diferencias entre varones y mujeres en el ámbito laboral. Parece, entonces, que la sociedad acepta como estado normal de las cosas el hecho de que sea la mujer la que renuncie a posibilidades profesionales para ocuparse de las obligaciones familiares mientras que del varón no se espera una conducta similar ni en la empresa ni en la propia familia (Tobío, 2005: 89-102). Las decisiones que se toman, desde este supuesto cultural, en las empresas y en las familias no hacen sino dar continuidad a este modo de comprender la distribución de las tareas familiares y las posibilidades de desarrollo profesional. 


\section{Estrategias de conciliación: diversidad de perspec- tivas y prioridades}

Los distintos sujetos implicados en el problema de la conciliación han ido desarrollando diferentes estrategias de respuesta según ha ido evolucionando el contexto familiar, empresarial, legislativo y cultural.

Las familias. En España, para muchas familias, conciliar significa todavía encontrar arreglos privados, en permanente revisión, frente a las demandas contradictorias del mundo del trabajo y de la esfera doméstica (Crespo y Moretón, 2010; Borrás, Torns y Morena, 2007). Entre estos «arreglos privados» se cuentan principalmente estos: la postergación de la maternidad; la disminución del número de hijos; el recurso a la ayuda de otros familiares, sobre todo de las abuelas, y la reducción de la jornada laboral, lo que suele llevar consigo la reducción de ingresos y de posibilidades de desarrollo de la carrera profesional.

En general, el peso de estas «estrategias» recae mayoritariamente en las mujeres. De acuerdo con diversos estudios, lo que más ayuda a las madres trabajadoras a hacer familia y empleo compatibles es, por este orden: la ayuda de su propia madre, la ayuda de su marido o de otros miembros de su familia, vivir cerca del trabajo o del colegio de los niños, la ayuda doméstica remunerada y los servicios que ofrece el centro escolar (ECFE, 2005). Según datos del INE, en 2010, en España, casi 700.000 personas redujeron su horario laboral para cuidar a un hijo menor de ocho años. El 85\% eran mujeres y el 15\% varones. Otras 260.000 personas tomaron excedencia a tiempo completo para atender a un hijo menor de ocho años, un 93\% de las cuales eran mujeres. Casi la mitad de las personas que piden estas excedencias no suelen reincorporarse después al empleo remunerado.

El discurso de las madres que trabajan a jornada parcial es, con frecuencia, contradictorio (Fernández y Tobío, 2005: 39-40). Por un lado, hay muchas mujeres que opinan que es una posibilidad interesante mientras los hijos son pequeños, e incluso manifiestan que, de hecho, trabajan más horas de las que querrían. Por otro lado, en cambio, manifiestan una cierta desconfianza hacia el trabajo parcial pues se percibe como más inseguro, menos remunerado y con peores condiciones en general.

La participación de los varones en las «estrategias familiares» de conciliación es creciente en estos últimos años pero aún muy escasa en términos comparativos. Aún cuando el modelo de corresponsabilidad se va imponiendo progresivamente en la mentalidad de la sociedad, las expectativas generalizadas en el ámbito laboral y la inercia de un modelo cultural pretérito sobre la distribución de tareas en el hogar actúan como frenos para el desarrollo de nuevas formas de convivencia familiar. 
Las empresas. El número de empresas públicas y privadas que ponen medidas de conciliación a disposición de sus empleados no ha hecho sino aumentar en los últimos años. Por una parte, están obligadas por los cambios legislativos al efecto. Por otra, responden poco a poco a una mentalidad social cada vez más favorable a la conciliación, y también a la creciente comprobación de que, si se organizan adecuadamente, las políticas de conciliación no afectan negativamente a la productividad sino más bien al contrario (IFREI, 2006; Mercader, 2008).

Las medidas que adoptan las empresas con mayor frecuencia son aquellas que persiguen los siguientes objetivos específicos (Meil y otros, 2010): flexibilizar el tiempo de trabajo mediante la adaptación de los horarios, el calendario o el turno de trabajo a las circunstancias personales; flexibilizar el lugar del trabajo, con medidas como el teletrabajo, las videoconferencias y otras tecnologías; conceder permisos adaptados a las necesidades reales de los empleados; ofrecer servicios como guarderías o centros de día; dar ayudas económicas por nacimiento de un hijo, seguros y otros beneficios extrasalariales de diversos tipos. En algunos sectores de actividad - por exigencias de la actividad, del servicio, de la organización de turnos u otras razones - existen barreras que hacen difícil la adopción de este tipo de medidas. Por su parte, en las empresas pequeñas y medianas existen menos recursos económicos y organizativos para implantar las fórmulas de conciliación que se observan en las empresas grandes (Chinchilla, 2007). Por todo ello, las medidas de conciliación se gestionan y desarrollan en función del contexto empresarial en que se precisan.

En cualquier caso, desde el punto de vista empresarial, las medidas de conciliación se presentan como fórmulas de flexibilización e innovación en políticas de recursos humanos, útiles para fidelizar a los empleados con alto valor añadido. Con estas medidas buscan beneficios para la empresa, como la mejora de la imagen corporativa; la mejoría del clima laboral; la reducción del absentismo, y, como consecuencia de todo ello, en último término, el aumento de la productividad.

La perspectiva política. Hasta mediados de los años noventa la Unión Europea enfocó el tema de conciliación desde la óptica de la igualdad de oportunidades entre varones y mujeres. En cambio, a partir de entonces lo ha situado en el contexto de la política de empleo (Eurofound, 2010). Las Estrategias Europeas de Empleo derivadas del Tratado de Lisboa propusieron como objetivo aumentar el empleo femenino. Desde entonces se han impulsado diversas políticas específicas de conciliación, basadas principalmente en dos tipos de actuaciones: servicios de atención a la vida diaria y permisos laborales para el cuidado de niños pequeños y de personas dependientes. En España, la Ley de Conciliación de la Vida Familiar y Laboral de Personas Trabajadoras (Ley 39/1999) incorporó a la normativa 
española las directivas europeas sobre permisos de maternidad, permisos parentales y trabajo a tiempo parcial. Desde entonces, una serie de medidas legislativas han contemplado de manera directa o indirecta diversos aspectos de las necesidades de conciliación, en particular, la Ley de Promoción de la Autonomía Personal y Atención a las Personas en Situación de Dependencia (Ley 39/2006) y la Ley para la Igualdad Efectiva de Mujeres y Hombres (Ley 3/2007).

En los últimos años hay un claro aumento de la atención que reciben los problemas de conciliación en las programas de los distintos partidos políticos. Estos apoyan distintos tipos de actuaciones según sus respectivas adscripciones ideológicas, con un mayor énfasis en la oferta de servicios públicos, por un lado, y en los permisos, la flexibilidad horaria y las ayudas fiscales, por otro (Fernández y Tobío, 2005: 21-24).

El propósito principal de todas estas propuestas políticas en materia de conciliación es estimular la incorporación de las mujeres al mercado de trabajo en condiciones de igualdad con los varones. Se espera que, en un breve plazo, la tasa de empleo femenino no sea inferior al $60 \%$ en ningún país europeo. Las razones que avalan esta meta son, básicamente, de tipo macroeconómico. Según la Comisión Europea, «las políticas de conciliación son la respuesta clave a los retos económicos y demográficos a largo plazo, y se deben exigir como medio para estimular el crecimiento» (Eurofound, 2010: 6). La perspectiva política para la conciliación es explícitamente una perspectiva funcional en la que priman las exigencias del sistema económico. Como comenta críticamente Torns (2011:8), el interés político en la cuestión de la conciliación no ha sido otro que el de lograr el aumento de disponibilidad laboral de la población ocupada, en particular de las mujeres, priorizando las necesidades de competitividad del sistema económico por encima de las necesidades de la población.

La perspectiva cultural. Las políticas de los poderes públicos tienen obviamente una dimensión cultural, en la medida en que promueven un determinado modelo de sociedad. Por eso, en la práctica, las políticas de conciliación se están convirtiendo en arena de disputa y confrontación no solo de distintas opciones políticas sino también de modelos alternativos de convivencia social y cultural.

En este contexto, avanzar medidas de conciliación significa tomar partido en una confrontación ideológica, según las consecuencias comprobadas o previsibles en valores como la igualdad social, la corresponsabilidad o la igualdad de género. Los partidos políticos y también múltiples organizaciones y actores de la sociedad civil son conscientes de que las políticas de conciliación sostienen determinados intereses y valores culturales. Así se comprende que el objetivo de las políticas de la UE sea la creación de una sociedad de «dos sustentadores» con 
el apoyo de servicios de cuidado garantizados por el Estado. Es un objetivo que sostiene y refuerza un modelo de convivencia en el que hombres y mujeres tienen empleo a tiempo completo y la atención y cuidado de niños pequeños y personas dependientes se organiza socialmente (social care).

Según las diferentes adscripciones ideológicas, estos cuidados se pueden organizar según criterios de mercado (postura liberal), como provisiones de servicios públicos (postura socialista) o según alguna combinación de ambas, económica y políticamente razonable y sostenible. Esta dependencia de un modelo ideológico es más explícita en algunos autores, como aquellos para quienes el objetivo de las políticas de conciliación ha de ser la «desmercantilización» y la «desfamiliarización» del sistema de bienestar de modo que sean los poderes públicos los que promuevan y garanticen un modelo de convivencia familiar basado en la autonomía personal en términos de igualdad (cfr. Campillo, 2010; Esping-Andersen, 2000). En esta propuesta, «desmercantilización» significa que el Estado debe garantizar el acceso a servicios de cuidado independientemente del nivel económico que se ocupe; es decir, que no es necesario ocupar un empleo remunerado para tener acceso al sistema de bienestar. «Desfamiliarización», análogamente, significa que el acceso a servicios de cuidado no debe depender de la situación familiar: se trata de que los individuos sean autónomos con respecto a sus familiares para acceder a servicios de cuidado, que deben ser garantizados por el Estado en términos de igualdad para individuos autónomos.

En sintesis. En las estrategias de conciliación confluyen diversos sujetos e intereses sociales, económicos, culturales y políticos. El riesgo de esta pluralidad es que la complejidad del problema sea tratada, en cada esfera, en términos de autorreferencialidad, según criterios funcionales que primen la eficacia en el logro bien de la competitividad empresarial, bien del rédito político, bien de la diseminación de un modelo cultural, a costa de los proyectos de vida de las familias mismas, que se encuentran cada vez más mediatizadas por condicionamientos sobrevenidos a su propio modo de existencia.

Uno de los problemas mayores que enfrentan las políticas de conciliación es precisamente esta definición en términos funcionales (y no relacionales) de las necesidades y alternativas disponibles para la armonización de las relaciones entre familia y trabajo. De hecho, lo que se echa en falta en las políticas actuales es una consideración explícita de lo que se pretende equilibrar (familia y trabajo) que evite la tendencia a la subordinación de ambas a criterios externos de carácter autorreferencial. Desde la perspectiva de la sociología relacional, autores como Pierpaolo Donati han tratado de establecer una consideración más acorde con la realidad y las necesidades de la conciliación. 


\section{La tensión familia-trabajo desde el enfoque relacio- nal de P. Donati}

\subsection{La familia en la sociología relacional}

El punto de vista desde el que se observan las relaciones familiares determina en gran medida la consideración y el modo en que se articula la problemática de la conciliación en términos laborales, políticos y culturales. Las perspectivas teóricas más frecuentes son, de hecho, el paradigma sistémico estructural y el paradigma individualista de la acción social. En el primero, que subraya el carácter objetivo, holístico y explicativo de la teoría, la familia es vista - desde un enfoque materialista (Marx) o positivista (Durkheim) - como una organización o institución creada por el sistema social. En el segundo paradigma, de carácter subjetivo y comprensivo, la familia se entiende como un grupo primario o un agregado de individuos unidos por lazos afectivos y sentimentales, o bien simplemente como un nombre, una convención o una representación mental.

El enfoque relacional propuesto por Donati $(1992 ; 2011)$ se distancia de ambas posturas y también de una posible «tercera vía» entendida como mezcla y compromiso entre estructuralismo e individualismo. Por el contrario, propone un paradigma alternativo, que se aleja tanto del materialismo y el positivismo propios de los enfoques sistémicos y estructuralistas, como del relativismo y el nominalismo propios de las teorías subjetivistas. Donati se suma de esta manera a la corriente de revitalización del realismo crítico $^{1}$, si bien entendido como un realismo relacional, analítico y no ingenuo, consciente del «doble nivel hermenéutico» desde el que se ha de observar críticamente la realidad, también relacional, que compone la vida social. Desde el punto de vista del realismo crítico, la familia se entiende como una relación social (mejor, una network o red de relaciones) que se corresponde con una realidad sui generis, de orden propio, si bien intangible e inmaterial, y también, por supuesto, contingente e incierta. Su carácter contingente, como el de toda relación, implica estar sometida a incertidumbre, y aunque la posibilidad de observación no es tangible no por ello deja de ser real.

La relación familiar tiene un carácter primordial para la sociedad, pues opera la transmisión cultural de generación en generación. En este sentido, es impres-

$1 \mathrm{El}$ realismo crítico es una corriente filosófica que sostiene que la realidad, si bien existe y es independiente de nosotros, no puede ser conocida de manera absoluta, sino que nuestro conocimiento de ella solo puede ser aproximado. La diferencia con el realismo ingenuo está en que este último considera que el ser humano conoce el objeto sin intermediarios, mientras que los realistas críticos afirman que el conocimiento se realiza a través de las cualidades sensibles del objeto y con una interacción observante-observado. Como posición espistemológica alternativa al individualismo y al estructuralismo está cobrando creciente importancia en diversas corrientes de teoría sociológica (cfr. Archer, Bhaskar, Collier, Lawson y Norrie, 1998; Maccarini, Morandi, Prandini 2011; Donati 2011). 
cindible para el avance en el proceso de civilización, ya que de hecho realiza el proceso de humanización y socialización del individuo, en que se constituyen las personas como «ser-en-relación». Por esta razón, Donati (2003: 25 y ss.) añade a la relación familia el adjetivo de «plena». La expresión «relación social plena» indica que la familia no solo está en el origen de la sociedad (en sentido filogenético y ontogenético) sino que la trasciende y va más allá, abarcando todas las dimensiones de lo humano. Ello significa que la familia no puede ser entendida fuera de un contexto que tiene componentes históricos, económicos, culturales y físicos, y también debe tener en cuenta las características psicológicas y biológicas de sus miembros. En definitiva, en Donati, «plena» (o también «total») significa que tiene en cuenta la multidimensionalidad del fenómeno social que constituye la familia y su carácter inagotable, porque engloba todas las dimensiones de la vida humana: social, psicológica, económica, religiosa, cultural, legal, política, y cualquier otra que pueda darse.

La forma y contenido de la relación familia se modifica por la continua e inevitable morfogénesis social, que le afecta tanto en la esfera de las relaciones externas como en la de las relaciones internas. Estas dos esferas se mantienen en continua interacción. Precisamente los cambios sociales y las transformaciones de la familia se deben a que los individuos, en su interacción, modifican los vínculos, las estructuras, los modos de «hacer familia». Así se explica, por ejemplo, según Donati, el paso de la familia extensa a la nuclear, o la emergencia de la familia relacional.

En la teoría donatiana, la familia es una matriz existencial que une a una pluralidad de individuos mediante relaciones afectivas y generativas y que da a la persona, a cada persona, su ubicación en el mundo. La familia existe a partir de la presencia de, al menos, una de sus dos relaciones básicas, que son la de pareja o conyugal y la de filiación.

La identidad («el genoma») de la familia está constituida por cuatro variables que circulan por la matriz relacional: sexualidad, generatividad, reciprocidad y don, siendo la más importante desde el punto de vista explicativo esta última, el don, que atribuye el sentido y la unión al conjunto. No es propio de la familia actuar solo por motivaciones sexuales, ni estar guiada exclusivamente por el objetivo de la procreación, ni cumplir un papel social de cumplimiento puramente normativo de derechos y obligaciones recíprocas. En la familia se integran todas estas dimensiones a través de la donación interpersonal.

La variabilidad familiar depende del peso o la importancia de cada una de estas dimensiones y de su mayor o menor integración. Es cierto que la sociedad actual presenta tendencias a la disgregación, y que, al hablar de relaciones sociales, 
siempre hay contingencia. La conexión entre las cuatro dimensiones varía según las épocas, y la separación podría darse de modo localizado y coyunturalmente, pero no es realista plantear una ruptura total y permanente; ello supondría la desaparición de la familia. A nivel empírico, Donati (2003:35ss) indica que estas dimensiones están más integradas en las formas de familia institucionalizadas y menos en las provisionales.

Algunos sociólogos hablan del amor como medio simbólico de intercambio en la familia, pero el término «amor» tiene una semántica ambigua y polivalente. Es necesario precisar más. El amor familiar incluye elementos como la confianza, la solidaridad o la reciprocidad, que se dan también en otras esferas informales. En la familia tiene su propia perspectiva: es un amor de don interpersonal y característico que construye, mediante la función de socialización de los hijos, las orientaciones altruistas que pueden luego ser trasladadas al resto de ámbitos sociales.

En una sociedad cada vez más tecnológica, artificial e impersonal, con problemas como el individualismo, la marginación, la soledad o la anomia social, la familia, como relación, es cada vez más decisiva para la calidad de vida social. Especialmente en las relaciones interpersonales, pero también en la convivencia civil solidaria y en la ética pública. La familia, con sus procesos socializadores, se hace imprescindible e insustituible para la convivencia y la orientación interpersonal y social hacia la dignidad humana. La familia genera un valor social añadido y proporciona bienes relacionales a la sociedad, en razón de sus mediaciones. Es, en sí misma, un bien relacional. «Bien relacional es un bien que solo puede ser producido y disfrutado por sus mismos usuarios y productores, mediante las relaciones que conectan a los sujetos participantes. El bien es relacional porque está/es la relación» (Donati, 1999: 123-124).

En definitiva, siguiendo la teoría genético-relacional de la familia podemos definir la familia como una realidad sui generis hecha de un específico bien relacional que va más allá de los individuos, una relación social plena que se da en una esfera de intimidad abierta, sujeta por tanto a un proceso de morfogénesis en su continua interacción con el exterior, y caracterizada por el don, la gratuidad y la reciprocidad entre los sexos y las generaciones.

\subsection{El trabajo visto desde la sociología relacional}

La industrialización trajo consigo un cambio en la concepción del trabajo, y también una subordinación de la persona a este. No hablamos solo de los cambios organizativos producidos por la división, la especialización del trabajo y la mecanización, sino también de algo más profundo. En un ambiente cada vez más 
técnico, artificial y burocrático, el trabajo se convierte en algo estresante que no facilita el desarrollo y la expresión de virtudes humanas. Una situación que ha favorecido una creciente deshumanización del trabajo. El problema social del trabajo generado por el capitalismo moderno aún no ha encontrado soluciones plenamente satisfactorias.

En la sociedad occidental actual se encuentran posturas contradictorias respecto al trabajo. Por un lado, hay una visión negativa que degrada el trabajo a actividad puramente instrumental, una prestación funcional destinada a aumentar los ingresos, la producción y el consumo. En esta visión, puramente economicista, el trabajo es un factor de producción más, que puede ser sustituido por la tecnología, y además, en esa sustitución se genera «tiempo libre». Por otro lado, y en sentido contrario, la exaltación del trabajo y la búsqueda de la realización personal (autorreferencial) en él ha llevado a otro modo de «esclavitud» de la persona al trabajo, propia también de los tiempos modernos.

La antítesis realización frente a alienación sigue siendo recurrente en los estudios sobre el trabajo. En el siglo xxI, y en un contexto de tránsito hacia una sociedad relacional, sin embargo, parece que algo está cambiando en el modo de concebir el trabajo: se tiene más consciencia de que el trabajo debe ser al servicio del hombre y no al contrario. Se valora más el tiempo libre que uno puede dedicar a la familia, amigos y a otras relaciones sociales, y el trabajo se piensa como una actividad necesaria, pero subsidiaria a la persona. El proceso de cambio, en medio de una crisis económica mundial, viene acompañado también de conflictos sociales, desempleo y precariedad. Además, el fenómeno de la globalización, que afecta a los mercados y a la economía, y los cambios en las nuevas técnicas, la informática y la tecnología, etc., abren nuevas posibilidades y hacen emerger un nuevo modo de entender la organización del trabajo, con un sentido, podemos decir, más relacional y más humano.

Aparecen así, en las dos últimas décadas, propuestas de rehumanización del trabajo, que contemplan una mayor autonomía, libertad y responsabilidad de la persona, y un sentido del trabajo no meramente instrumental. Dentro de estas propuestas están la participación y la autogestión de los trabajadores, la coparticipación multi-staker, proyectos de calidad del trabajo y otras que, aunque en principio incluyen aspectos más de tipo organizativo y material que relacional, pueden ser el camino hacia la «emergencia» de una nueva concepción del trabajo como algo propio de lo humano, en el sentido relacional donatiano.

Revaluar el trabajo humano implica, ante todo y desde el punto de vista social, distinguir y separar el trabajo del producto del trabajo. Significa también que el trabajo no debe atender solo exigencias económicas de productividad, com- 
petitividad, rendimiento, eficiencia, etc., sino también a exigencias humanas, de relacionalidad social con los otros: compañeros, clientes, proveedores, jefes... La idea de fondo es la de un trabajo que mejore la calidad de vida de la persona, entendida esta no solo como bienestar material (welfare) y psicológico (well-being), sino también y principalmente como «vida buena» en sentido moral (Donati, 2005b). La reconfiguración del trabajo como actividad propiamente humana es la base ética fundamental de la empresa, que debe contemplar aspectos tales como la motivación del trabajador, su libertad, su capacidad de iniciativa y el desarrollo de su vocación profesional.

En esta línea, Donati (2005a) propone el «contrato relacional de trabajo», como expresión de la regulación de una relación que no afecta solo a dos partes, sino también a la comunidad. Cada vez más, el trabajo se vincula a circuitos de intercambio que implican el bien común de una red de productores, consumidores, trabajadores, entorno local, y de la sociedad en general.

En Donati, el trabajo no es solo la "prestación a cambio de un salario», sino «toda actividad interpersonal orientada a un objetivo socialmente útil de cuya realización depende la obtención de recursos para vivir por parte de quien lo cumple y de quien lo recibe» (Donati, 2001: 253). Por tanto, el concepto incluye tanto el trabajo que produce bienes y servicios para un cliente impersonal y con un valor de cambio o precio, como el que produce bienes y servicios para personas concretas en un contexto determinado, como en la familia o en una asociación de voluntariado, por ejemplo, donde no hay precio, sino valor de uso. En esta concepción, destaca el carácter ético no utilitarista, sino finalista, del trabajo.

Donati habla del trabajo como un «hecho social total», en el sentido de que trasciende la mera prestación instrumental para alcanzar un significado social, moral, económico, utilitario, jurídico y afectivo. Desde su perspectiva, la organización del trabajo debe combinar la libertad de los sujetos y su responsabilidad, buscando sinergias y cooperación entre los roles profesionales, y no solo competitividad. Además, la realización de la persona se ha de entender no de modo autorreferencial, sino en la relación con los otros, en todos los ámbitos vitales, familia, escuela, comunidad local y empresa, de manera equilibrada e integrada. La autorrealización de la persona como «ser-en-relación» con los otros da valor al trabajo como bien común, o bien relacional, en la terminología donatiana. 


\section{La conciliación trabajo-familia en clave relacional: un punto de partida}

La sociedad industrial capitalista estableció la diferenciación funcional entre el trabajo y la familia, separando netamente el ámbito de vida de la familia, en la esfera privada, lugar de los afectos y de las relaciones humanas intersubjetivas, del ámbito del trabajo, público, impersonal y utilitarista. El conflicto aparece precisamente por la separación de estos dos ámbitos que se rigen por códigos simbólicos diferentes que, en principio, se perciben como incompatibles: en la familia funciona el código del amor y del don, en el trabajo funciona la lógica de mercado y los criterios económicos.

En la sociedad postindustrial aparece una nueva sensibilidad: la familia empieza a considerar el tiempo como factor fundamental para la calidad de vida. El tiempo y su disponibilidad se presentan en el contexto familiar como la clave del bienestar y de la conciliación. En muchas empresas, aunque no en todas, aparece también una nueva sensibilidad social, que contempla a las familias de sus trabajadores.

Es precisamente en este tránsito hacia una nueva sociedad donde se hace necesaria la óptica relacional. En este enfoque, familia y trabajo no resultan incompatibles, sino interdependientes. Frente a la diferenciación funcional, Donati (2005a) propone una diferenciación relacional en la que ambas esferas, familia y trabajo, no funcionen ya por autorreferencia, sino por relación entre síł la familia pertenece al ámbito privado, pero no por ello está privada de funciones sociales; el trabajo pertenece al ámbito económico y de mercado, pero no por ello carece de sentido humano. En el enfoque relacional, Donati propone contemplar el problema desde la óptica de la subsidiariedad. Si se da prioridad absoluta al trabajo, considerando a la persona de modo individual y no relacional (familiar), ello supone la destrucción del capital social primario básico para la sociedad, que es el capital social familiar.

Una política de conciliación en clave relacional debe tener en cuenta a todos los actores sociales implicados: empresas, sindicatos, gobierno central y local, asociaciones del tercer sector y del privado social y, especialmente a las asociaciones familiares. Ello debe ser así, precisamente, porque se trata de una relación, la de trabajo y familia que, bien gestionada, supone una fuente básica de generación de capital social.

La conciliación vista de este modo se enmarca en el modelo de welfare que propone Donati (2005a), que es un welfare societario plural (relacional), caracterizado porque las instituciones públicas asumen un papel subsidiario en la 
promoción de la organización societaria (multi-stakeholder). En este modelo, la familia (o la mujer) no tiene simplemente la condición de asistida por el Estado, sino que también es productora y consumidora del propio bienestar. Este modelo confiere a la familia el papel de sujeto decisorio (empowerment). La relación trabajo-familia no puede ser gestionada entre Estado y mercado dejando fuera a las familias, a las asociaciones y a la comunidad en la que se desenvuelven. Donati propone una política que contemple la conciliación en términos de reciprocidad en las relaciones antes que en términos de meros intercambios o intereses económicos.

Aunque las políticas de conciliación son diferentes según los países, en general, en Europa dominan las políticas entendidas como un modo de aumentar el empleo femenino, actuar contra la discriminación y romper el llamado «techo de cristal». Ello se busca a través del sistema Estado-mercado, al que se le atribuye el papel de propulsor del workfare. Workfare es un término que se refiere a las medidas políticas que obligan a un individuo a asumir una ocupación en una actividad prevista por el propio gobierno, o a hacer alguna actividad para la reinserción en el mercado de trabajo con el fin de poder disfrutar de los derechos del welfare. En ese modelo, la relación sinérgica entre trabajo y familia es vista principalmente, por no decir únicamente, desde la óptica de la capacidad y productividad del sistema.

La estrategia subsidiaria horizontal que propone Donati valora en términos de reciprocidad la relación entre el trabajo y la familia, dando prioridad a esta última, y sin que ello suponga una devaluación del trabajo, sino más bien una humanización de este, un aumento de su valor humano. El Estado se entiende aquí como agente subsidiario de la sociedad civil. En este enfoque las medidas de conciliación son definidas principalmente por el complejo empresas-familiastercer sector. El papel del Estado es intervenir allí donde sea necesario para la promoción de la relación trabajo-familia. La sinergia es vista desde la óptica de la comunidad, del welfare comunitario. Lo que se busca es calidad de vida y bienestar a nivel comunitario, a partir de una relación equilibrada entre familia y trabajo. Con esta estrategia se abandona definitivamente la lógica funcional de la autorreferencialidad.

En este entramado societario, la empresa debe contemplar a la familia no solo como suministradora de mano de obra y como consumidora, sino como fuente de motivación y capacidad de trabajo, y proveedora ya no de recursos humanos, sino de capital humano y de capital social primario.

Las medidas conciliadoras más habituales de las empresas - como permisos de trabajo por motivos familiares, servicios de guardería o escuela infantil, 
ayudas para familiares discapacitados, trabajo a tiempo parcial, flexibilidad en los horarios de entrada y salida, teletrabajo, banco de horas, trabajo a turnos, cambios organizativos orientados a la paridad de género, buenas prácticas de sensibilización en la empresa sobre los derechos de las mujeres, asesoramiento y consejo a las mujeres para orientar su carrera profesional o formación específica para mujeres - son, sin duda, muy positivas, con carácter general, pero en la práctica se toman aún en función de razonamientos de utilidad y productividad y no de bienestar real, cooperación y confianza. De ordinario prevalecen la óptica de género, la de empresa y las consideraciones de utilidad contingente antes que la producción de un bien relacional entre el trabajo y la familia. Como advierte Donati (2005a), no hay aún una cultura relacional de la conciliación.

\section{Consideraciones finales: reflexiones y propuestas}

En el fondo del enfoque relacional está la idea de que la conciliación no debe reducirse a unas medidas empresariales o a un instrumento político cuyo objetivo sea aumentar la tasa de empleo femenina y demandar a los varones un compromiso y una participación mayores en las tareas domésticas. Esto es importante sin duda, pero reduccionista. La relación entre trabajo y familia es algo mucho más complejo.

El enfoque relacional opera desde la óptica del capital social. El problema debe ser visto y afrontado por un conjunto de actores (empresa, trabajador, sindicatos, familia, asociaciones familiares, Estado, comunidad local, etc.), de modo que no se reduzca a una mera negociación entre dos partes, sino que sea contemplado como parte de una red, entramado o sistema de relaciones de cooperación y reciprocidad. La conciliación, vista de este modo, actúa también en la regeneración del capital social familiar por encima de su simple consumo. Hoy muchos estudios tratan sobre la pérdida de capital social en la sociedad, pero pocos analizan la familia como fuente de capital social primario. Muchos de los problemas sociales actuales se deben precisamente al debilitamiento social de la familia.

En la perspectiva donatiana para una conciliación relacional del trabajo y la familia, se propone como instrumento práctico el llamado contrato relacional, que debe incluir aquello que la modernidad ha separado: el tiempo de trabajo remunerado y el tiempo de trabajo no remunerado familiar, es decir, un contrato que no solo contemple la prestación laboral, sino también aquellas relaciones sociales y familiares que hacen posibles y rentables las prestaciones. Por eso, en línea con la idea de la governance societaria, sería deseable la figura de un delegado o representante de las asociaciones familiares en la empresa, que vele por los intereses de 
las familias de los trabajadores y que funcione de modo análogo o similar a como lo hacen los representantes o delegados sindicales.

La conciliación entre trabajo y familia es un asunto complejo para el que no se pueden dar soluciones estandarizadas, pero Donati (2005a: 438-439) sí propone algunos principios guía como criterios para la evaluación y selección de medidas específicas adaptadas a cada contexto familiar y laboral. Son los siguientes:

Libertad de elección y paridad de oportunidades para conseguir una relación satisfactoria entre familia y trabajo profesional. Los adultos deben poder distribuir y armonizar la actividad laboral o profesional y la actividad familiar según sus necesidades personales y familiares. Libertad de elección e igualdad de oportunidades son una expresión concreta de la diversidad de formas de vida.

Igualdad de derechos entre varones y mujeres. Las medidas y la normativa de conciliación deben tener en cuenta el interés de todos los miembros de la familia, considerando de modo específico la edad, el género y la fase del ciclo de vida que está atravesando cada uno.

Gestión del tiempo y coordinación de los horarios por parte de la autoridad local. Las medidas y la normativa conciliatoria de las instituciones y organizaciones locales deben tener en cuenta las necesidades específicas de la familia en lo que afecta a la programación de su vida cotidiana (horarios de apertura y de cierre, servicios de transporte público, etc.)

Estructura de apoyo a la familia, principalmente a través del establecimiento de contratos relacionales y de la acción de las asociaciones familiares. La responsabilidad de los servicios de apoyo no puede ser delegada a las familias (a cada familia) o a miembros individuales de las familias, sino que tiene que estar gestionada por organismos intermediarios (sindicatos, asociaciones familiares, organizaciones de privado social,...). Las medidas y normativas de conciliación, tanto de las instituciones político-administrativas como de las organizaciones económicas, deben crear una estructura social adecuada que facilite la conciliación entre la vida laboral y la familiar para cada caso particular: infraestructura para la guarda y cuidado de niños pequeños, servicios domiciliarios, etc.

Las medidas políticas conciliatorias deben tener efectos estables en el tiempo.

La conciliación, además, no debe limitarse a reivindicaciones de tiempo. El punto central debe estar en un nexo de libertad y responsabilidad tanto individual como colectiva de la organización del trabajo, que permita el desarrollo de compromisos razonables.

En definitiva, las soluciones al problema de la conciliación entre trabajo y familia se deben pensar en términos de flexibilidad, tanto por parte de la organización del trabajo profesional como por parte de la familia. Ahora bien, flexibilidad 
no significa mera adaptabilidad, sino que las vías y el modo de conciliar deben ser asumibles tanto en la familia como en el trabajo. Para la política de conciliación, según la propuesta de Donati, se debe adoptar una nueva relacionalidad recíproca entre trabajo y familia mediante una estrategia subsidiaria en la que la sociedad civil sea efectivamente protagonista de un nuevo modelo de welfare societario. Esta subsidiariedad debe atribuir la dignidad que corresponde a la familia y al trabajo y debe armonizar sus contribuciones específicas al desarrollo civil de la sociedad. Ello implica reconocer las funciones sociales de la familia como insustituibles y como prioritarias desde el punto de vista existencial.

\section{Bibliografía}

Albert, R.; Escot, L.; Fernández, J. A. y Palomo, M. T. (2010). Las políticas de conciliación de la vida familiar y laboral desde la perspectiva del empleador. Problemas y ventajas para la empresa, en Cuaderno de Trabajo 02/2010 de la E.U. Estadística, Universidad Complutense de Madrid.

Archer, M., Bhaskar, R., Collier, A., Lawson, T. y Norrie, A. (1998). Critical Realism: Essential Readings. London: Routledge.

Borrás, V;; Torns, T.; Morena, S. (2007). «Las políticas de conciliación: políticas laborales versus políticas de tiempo», Papers, 83: 83-96.

CAmpillo, I+ (2010). «Políticas de conciliación de la vida laboral y familiar en los regímenes de bienestar mediterráneos: los casos de Italia y España», Política y Sociedad, 47(1): 189-213.

Cardona, M. B. (2009). Medidas de conciliación de la vida personal, laboral y familiar. Hacia un respeto igualitario de las reformas parentales. Albacete: Bomarzo.

Chinchilla, N. (2007). Ser una empresa familiarmente responsable. ¿Lujo o necesidad? Madrid: Pearson, Prentice Hall.

Consejería de Empleo y Mujer de la Comunidad de Madrid. (2005). Encuesta sobre conciliación de la vida laboral y personal de la población de la Comunidad de Madrid: informe de resultados. Madrid: TNS Demoscopia.

Crespo, M. y Moretón, F. (2010). Conciliación de trabajo y familia. Las claves del éxito: eficiencia empresarial e implicación masculina. Madrid: Colex.

Donati, P. (1992). Teoria relazionale della società. Milano: FrancoAngeli.

- (1999). La ciudadanía societaria. Granada: Universidad de Granada.

- (2001). Il lavoro que emerge. Prospettive del lavoro come relazione sociale in un'economia dopomoderna. Torino: Bollati Boringhieri.

- (2003). Manual de sociología de la familia. Pamplona: Eunsa. 
- (2005a). Famiglia e lavoro: dal confitto a nuove sinergie. Milano: San San Paolo.

- (2005b). Il lavoro e la persona umana. Comunicación presentada en el 3er. Simposio Europeo dei Docenti Universitari, Roma.

- (ed.) (2007). Famiglie e bisogni sociali: la frontiera delle buone prassi. Milano: FrancoAngeli.

- (2011). Relational Sociology. A new paradigm for the social sciences. London: Routledge.

EBTP-European Business Test Panel (2009). The importance of familiyfriendliness for business development. Disponible en $<$ http://ec.europa.eu/yourvoice/ebtp/consultations/family/report_en.pdf> (Fecha de consulta 11-092012).

ECFE (2005). Estudio sobre la conciliación de la vida familiar y la vida laboral: situación actual, necesidades y demandas, Observatorio para la Igualdad de Oportunidades entre mujeres y hombres. Madrid: Instituto de la Mujer, Ministerio de Trabajo y Asuntos Sociales.

Esping-Andersen, G. (2000). Fundamentos sociales de las economías postindustriales. Barcelona: Ariel.

Eurofound (European Foundation for the Improvement of Living and Working Conditions). (2010). Family Life and Work. Second European Quality of Life Survey. Luxembourg: Office for Official Publications of the European Communities.

Eurostat (2005). «Gender gaps in the reconciliation between work and family life», Statistics in focus, Populations and Social Conditions, 4.

Fernández, J.A. y Tobío, C. (2005). Conciliar las politicas familiares y laborales: politicas y prácticas sociales, en Documento de trabajo 79/2005, Fundación Alternativas.

Hein, C. (2006). Conciliar el trabajo y las responsabilidades familiares. Ideas prácticas de la experiencia global. Informes OIT. Madrid: Ministerio de Trabajo y Asuntos Sociales.

IFREI (2006). Análisis sectorial de las políticas de conciliación. Barcelona: Centro Internacional Trabajo y Familia IESE. Disponible en <http://www.iese.edu/ research/pdfs/ESTUDIO-41.pdf> (Fecha de consulta 11-5-2012)

INE (2010). «Encuesta de Población Activa (EPA). Módulo sobre conciliación entre la vida laboral y la familiar» (<www.ine.es $>$ ).

INE (2011). «Encuesta de Empleo del Tiempo 2009-2011» (<www.ine.es>).

López, A.y Acereda, A. (2007). Entre la familia y el trabajo. Realidades y soluciones para la sociedad actual. Madrid: Narcea. 
Maccarini, A.; Morandi, E. y Prandini, R. (2011). Sociological Realism. London: Routledge.

Meil, G.; García, C.; Luque, M. Á. y Ayuso, L. (2010). El desafío de la conciliación de la vida privada y laboral en las grandes empresas. Madrid: Universidad Autónoma de Madrid.

Mercader, J. R. (2008). Productividad y conciliación en la vida laboral y personal. Análisis de situación y propuestas para el futuro. Madrid: Fundación Telefónica.

Montoya i BarberÁ, J. (2007). «Papel de la acción sindical y la negociación colectiva en las políticas de conciliación», en López, A. y Acereda, A. (coords.). Entre la familia y el trabajo. Realidades y soluciones para la sociedad actual, Madrid: Narcea.

OECD (2007): Babies and Bosses. Reconciling work and family life. A synthesis of Findings for OECD Countries. Paris: Organisation for Economic Cooperation and Development.

Товío, C. (2005). Madres que trabajan. Dilemas y estrategias. Madrid: Cátedra.

Torns, T. (2011). «Conciliación de la vida laboral y familiar o corresponsabilidad: ¿el mismo discurso?», Observatori per a la igualtat UAB, RIDEG, 1, 5-13. 